\title{
Ferroelectricity in a one-dimensional organic quantum magnet
}

\author{
Fumitaka Kagawa $^{1 \star}$, Sachio Horiuchi ${ }^{2}$, Masashi Tokunaga ${ }^{3}$, Jun Fujioka' and Yoshinori Tokura ${ }^{1}, 2,4,5$
}

In magnetically controllable ferroelectrics ${ }^{1-3}$, electric polarization is induced by charge redistribution or lattice distortions that occur to minimize the energy associated with both the magnetic order and interaction of spins with an applied magnetic field. Conventional approaches to designing materials that exploit such spin-mediated behaviour have focused mainly on developing the cycloidal spin order ${ }^{4,5}$, and thereby producing ferroelectric behaviour through the so-called antisymmetric Dzyaloshinskii-Moriya interaction ${ }^{6-8}$. However, engineering such spin structures is challenging. Here we suggest a different approach. Direct measurements of magnetic-field-dependent variations in the polarization of the one-dimensional organic quantum magnet, tetrathiafulvalene-p-bromanil, suggest a spin-Peierls instability has an important role in its response. Our results imply that one-dimensional quantum magnets, such as organic charge-transfer complexes, could be promising candidates in the development of magnetically controllable ferroelectric materials.

A straightforward guideline for designing spin-driven ferroelectricity might be to use the symmetric interaction, simply because it is more dominant in ubiquitous magnets, rather than the antisymmetric exchange interaction (that is, the Dzyaloshinskii-Moriya interaction). In this context, the up-up-down-down ( $\uparrow \downarrow \downarrow)$ magnetic order on the alternating (ABAB) atom sites was proposed as a promising candidate for symmetric-exchange-driven ferroelectricity ${ }^{9}$; this prediction has actually been confirmed in the frustrated Ising chain system, $\mathrm{Ca}_{3} \mathrm{Co}_{2-x} \mathrm{Mn}_{x} \mathrm{O}_{6}(\mathrm{~A}=\mathrm{Co}$ and $\mathrm{B}=\mathrm{Mn})^{10}$, and the $f-d$ spin-coupled state of $\mathrm{GdFeO}_{3}(\mathrm{~A}=\mathrm{Gd}$ and $\mathrm{B}=\mathrm{Fe})^{11}$. Moreover, there is a theoretical argument that symmetric-exchange-driven ferroelectricity can host potentially large polarization ${ }^{12}$. This recent progress is prompting a pursuit of not only materials showing the $\uparrow \uparrow \downarrow \downarrow$ order but also other clear-cut examples of symmetric-exchange-driven ferroelectricity.

In the same way as one-dimensional (1D) metals with inherent lattice instability (Peierls instability) ${ }^{13}$, 1D Heisenberg spin-1/2 quantum magnets possess an instability to form a dimer-singlet state because of the energy gain of symmetric exchange $^{14,15}$. This is known as the spin-Peierls instability, a textbook example of spin-lattice coupling. Therefore, the spin-Peierls instability with alternating $\mathrm{ABAB}$ spin sites can host a polar singlet-dimer and hence is expected to provide a new mechanism for magnetically controllable ferroelectrics of symmetric-exchange origin. The organic charge-transfer salt TTF-BA (tetrathiafulvalene- $p$-bromanil) has been proposed as a possible candidate of this new class $^{16}$ of material, namely a ferroelectric spin-Peierls material; nevertheless, direct observations of ferroelectricity, that is, electric-field reversal of polarization, have yet to be accomplished in TTF-BA. Furthermore, the prominent magnetoelectric effect is anticipated in the possible ferroelectric spin-Peierls phase because of the magnetic-field effect on the spin-Peierls transition, yet this is also unexplored. In this work, using successfully synthesized large crystals of TTF-BA (see the Methods section), we demonstrate both the ferroelectric properties and their magnetic-field modification and argue a new feature of the ferroelectric spin-Peierls transition.

According to the structural analysis of TTF-BA (ref. 17), the space group changes from $P \overline{1}$ (nonpolar) to $P 1$ (polar) below $T_{\mathrm{c}}$ $(\approx 53 \mathrm{~K})$, and a $1 \mathrm{D}$ polar stack is realized through the dimer-singlet formation of the ionic TTF donor $\left(\mathrm{D}^{+}\right)$and BA acceptor $\left(\mathrm{A}^{-}\right)$ molecules, as schematically shown in Fig. 1a. The real crystal structure (Fig. 1b) consists of two $\mathrm{D}^{+} \mathrm{A}^{-} \mathrm{D}^{+} \mathrm{A}^{-} \ldots$ mixed stacks (' $\alpha$ stack' and ' $\beta$ stack' along the $a$ and $b$ axes, respectively), and the dimerization occurs in both stacks ${ }^{17,18}$. Note that unlike the prototypical neutral-ionic transition system TTF- $p$-chloranil (ref. 19), TTF and BA molecules in TTF-BA are almost ionic in a whole temperature region (the ionicity is $\sim 0.95$; ref. 18 ); thus, the $\mathrm{D}^{+} \mathrm{A}^{-}$stack can be regarded as a $1 \mathrm{D}$ Heisenberg chain with spin$1 / 2$. In fact, TTF-BA undergoes a paramagnetic-to-non-magnetic transition at $T_{\mathrm{c}} \approx 53 \mathrm{~K}$ (Fig. $\left.1 \mathrm{c}\right)^{18}$, being consistent with the singlet formation in the 1D Heisenberg chain involving the spin-Peierls instability. The $\mathrm{D}^{+} \mathrm{A}^{-}$-dimerized stack is also manifested by the steep increase below $T_{\mathrm{c}}$ in the spectral weight of $a_{g}$ mode activity at $1,422 \mathrm{~cm}^{-1}$ probing the local $\mathrm{D}^{+} \mathrm{A}^{-}$dimerization ${ }^{18}$ (Fig. 1d). From the temperature dependence of spin susceptibility, the magnitude of the spin gap in the dimerized phase is estimated to be $\sim 230 \mathrm{~K}$ (Fig. 1c and Supplementary Fig. S1), which is clearly larger than $T_{\mathrm{c}}$. This means that the condensation energy of the dimerized phase originates mainly from the singlet formation. Therefore, the magnetism-lattice coupled phase transition at $53 \mathrm{~K}$ can be regarded as the spin-Peierls transition.

The actual dimerization is not necessarily face-to-face ${ }^{17}$, and thus the local polarization induced by each dimerized stack may deviate from the original stacking direction. To estimate the polarization direction, we calculated local polarizations by using a classical point-charge model, which is the first approximation in the case of an ionic crystal such as TTF-BA, and found that the dimerization pattern may result in total polarization $P$ approximately along the $b$ axis (slightly inclined towards the $a$ axis, see Supplementary Fig. S3 and Table S1). In fact, a sharp enhancement of the dielectric constant $\left(\varepsilon_{1} \approx 210\right)$ is observed at $\sim 53 \mathrm{~K}$ along the $b$ axis (Fig. 1e). In contrast with the blurred anomaly reported for the polycrystalline sample ${ }^{20}$, this clear feature is indicative of a ferroelectric transition. We also observed a $\varepsilon_{1}$ peak of $\sim 24$ along the $a$ axis, with no enhancement along the

\footnotetext{
${ }^{1}$ Multiferroics Project, ERATO, Japan Science and Technology Agency (JST), c/o Department of Applied Physics, University of Tokyo, Tokyo 113-8656, Japan, ${ }^{2}$ National Institute of Advanced Industrial Science and Technology (AIST), Tsukuba 305-8562, Japan, ${ }^{3}$ Institute for Solid State Physics, University of Tokyo, Kashiwa, 277-8581, Japan, ${ }^{4}$ Department of Applied Physics, University of Tokyo, Tokyo 113-8656, Japan, ${ }^{5}$ Cross-Correlated Materials Research Group (CMRG), RIKEN, Advanced Science Institute, Wako 351-0198, Japan. *e-mail: kagawa@erato-mf.t.u-tokyo.ac.jp.
} 


$$
\text { High-temperature phase }
$$

Low-temperature phase

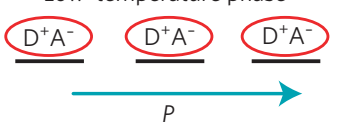

b

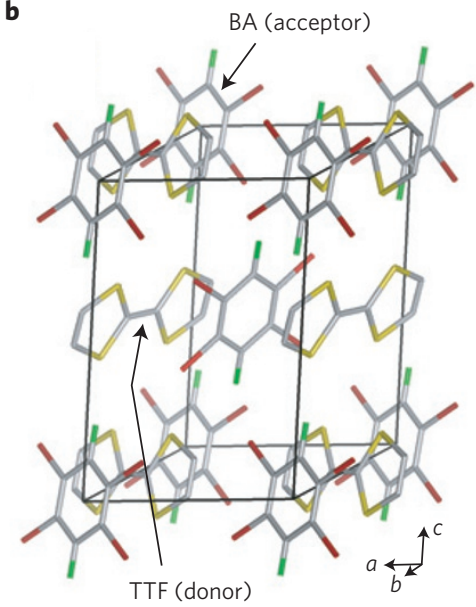



Figure 1 | Ferroelectric spin-Peierls transition in TTF-BA. a, Schematic structure of ionic donor $\left(D^{+}\right)$and acceptor $\left(A^{-}\right)$mixed stacks in high- and low-temperature phases of TTF-BA. The arrow, underline and ellipsoid represent spin-1/2, a dimer and a singlet state, respectively. $P$ denotes electric polarization. b, Crystal structure of TTF-BA. c-f, Temperature dependence of spin susceptibility (c), normalized spectral weight of the $a_{g}$ mode at $1,422 \mathrm{~cm}^{-1}$ as a measure of local $\mathrm{D}^{+} \mathrm{A}^{-}$dimerization (d), dielectric constant at $10 \mathrm{kHz}(\mathbf{e})$ and spontaneous polarization (f) along the $b$ axis. In $\mathbf{c}$, the spin susceptibility at $1 \mathrm{~T}$ is presented after subtracting both the estimated core diamagnetism $\left(\sim-2.2 \times 10^{-4}\right.$ e.m.u. $/$ mol f.u. $)$ and the Curie contribution discernible below $20 \mathrm{~K}$. The blue curve in $\mathbf{c}$ is the activation-type fitting (Supplementary Fig. S1), $\chi_{\mathrm{spin}} \sim \exp \left(-\Delta_{\mathrm{S}} / k_{\mathrm{B}} T\right.$ ), with the spin gap $\Delta_{\mathrm{S}}(\approx 230 \mathrm{~K}$ ). The temperature dependence of the $a_{g}$ mode spectra is shown in the inset of $\mathbf{d}$; see also Supplementary Fig. S2.

$c$ axis (Supplementary Fig. S4). This overall dielectric behaviour including the dielectric anisotropy is at least qualitatively consistent with our expectation. Below $53 \mathrm{~K}$, the spontaneous polarization $P$ was actually observed along the $b$ axis by pyroelectric current measurements (Fig. 1f). More directly, a clear $P-E$ hysteresis loop is also obtained (Fig. 2). The magnitudes of $P$ agree well except for around $43 \mathrm{~K}$ (Fig. 2, inset), demonstrating unambiguously that the low-temperature phase is not only composed of polar singlet-dimers but is also ferroelectric with reversible spontaneous polarization. The polarization at the lowest temperature is $\sim 0.15 \mu \mathrm{C} \mathrm{cm}^{-2}$, which is larger than the representative cycloidalspin-based multiferroics, $\mathrm{TbMnO}_{3}\left(\sim 0.08 \mu \mathrm{C} \mathrm{cm}^{-2}\right.$; ref. 1$)$.

Next, we investigate the potential magnetoelectric coupling in the ferroelectric dimer-singlet phase. If the $\mathrm{D}^{+} \mathrm{A}^{-}$-dimerized ferroelectricity results from the spin-Peierls instability as postulated, the polarization should vanish when the singlet state is suppressed by a magnetic field. The $M-H$ curves at various temperatures are shown in Fig. 3a. Below $T_{c}(\approx 53 \mathrm{~K})$ the characteristic nonlinearity is observed, whereas it is absent above $T_{c}$; moreover, the susceptibility (that is, the slope of $M-H$ curve) is larger in the high-field phase than that in the low-field phase. These characteristics indicate a breakdown of the singlet state at some critical field, $H_{\mathrm{c}}(T)$. More importantly, this singlet suppression is accompanied simultaneously by the disappearance of $\mathrm{D}^{+} \mathrm{A}^{-}$dimerized ferroelectricity: as shown in Fig. 3b, the polarization at 50 and $52 \mathrm{~K}$ is almost extinguished around $H_{\mathrm{c}}(T)$, indicating that the paraelectric uniform stack is restored. At lower temperatures, $P$ does not vanish up to $\sim 56 \mathrm{~T}$; yet the partial suppression is still obvious at $40 \mathrm{~K}$. Therefore, we conclude that the $\mathrm{D}^{+} \mathrm{A}^{-}$-dimerized

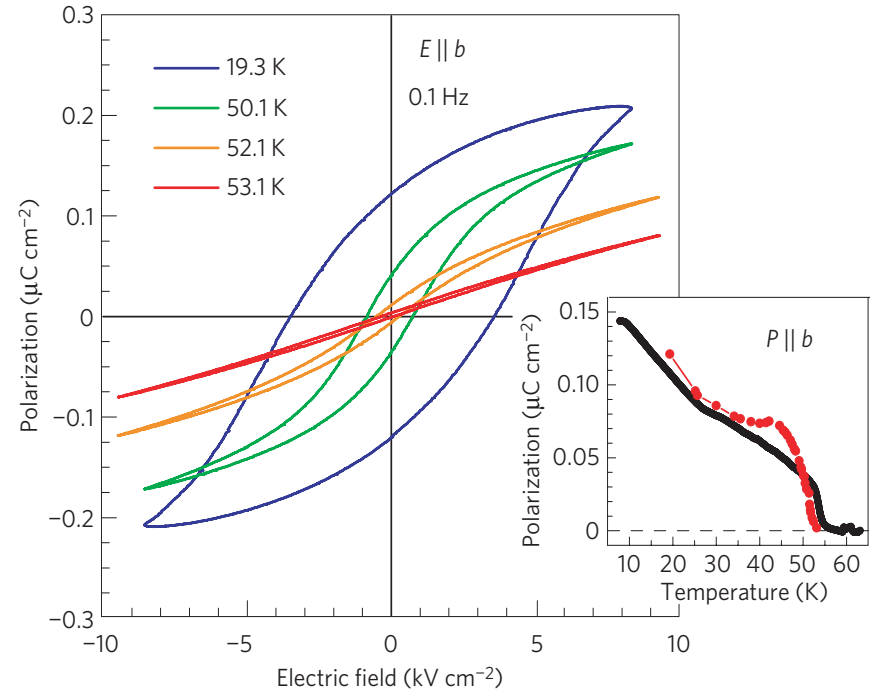

Figure 2 | $\mathbf{P}-\boldsymbol{E}$ hysteresis loop along the $\boldsymbol{b}$ axis. The measurement was done at $0.1 \mathrm{~Hz}$. Inset: Comparison between the temperature dependence of remnant polarization obtained in the $P-E$ hysteresis loop and that obtained in the pyroelectric measurement (Fig. 1f).

ferroelectric transition is primarily due to the magnetic energy gain, that is, spin-Peierls instability; nevertheless, it is possible that other mechanisms (for example, long-range Coulomb interaction) assist the ferroelectric spin-Peierls state. As far as we know, TTF-BA is 
a

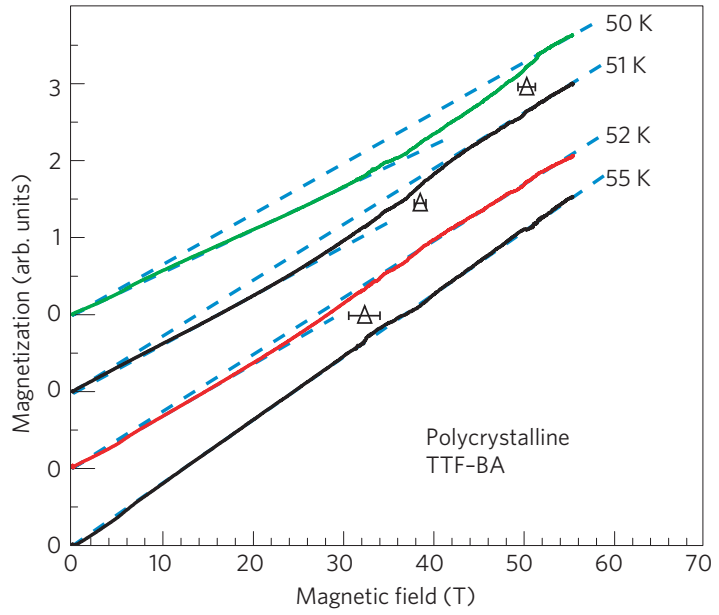

b

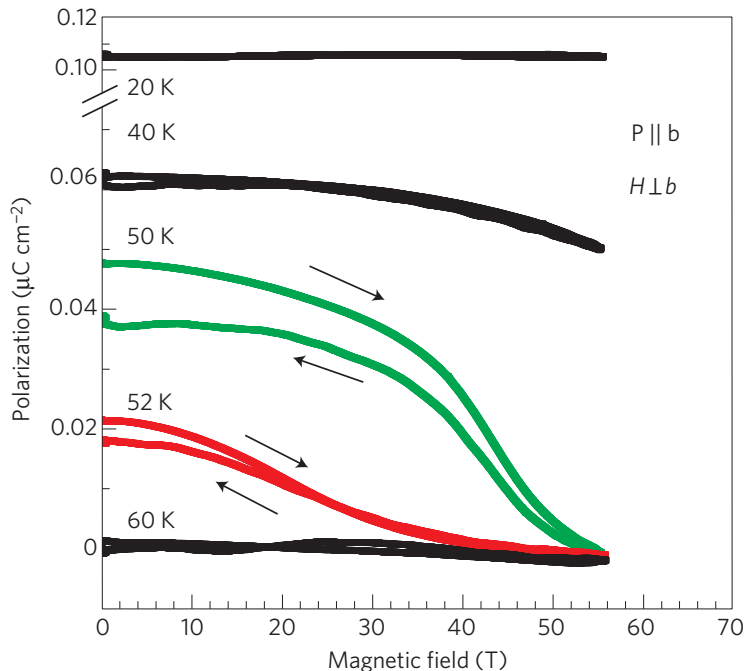

Figure 3 | Magnetic-field-induced transition from the ferroelectric nonmagnetic phase to the paraelectric paramagnetic phase. a, $\mathrm{M}-\mathrm{H}$ curves of TTF-BA (assembly of randomly oriented small crystals) at various temperatures. The triangles indicate the critical field where $\mathrm{d} M / \mathrm{d} H$ becomes a maximum. The error bars represent the numerical ambiguity of the $\mathrm{d} M / \mathrm{dH}$ maximum. $\mathbf{b}$, Magnetic-field dependence of electric polarization $(\| b)$ under $H(\perp b)$ at various temperatures in TTF-BA.

the first material in which the ferroelectric spin-Peierls state and its prominent magnetoelectric coupling have been experimentally identified. Note that in other prototypical spin-Peierls systems such as TTF-CuS $\mathrm{C}_{4}\left(\mathrm{CF}_{3}\right)_{4}$ (ref. 21) and $\mathrm{CuGeO}_{3}$ (ref. 22), the 1D Heisenberg chain consists of a single-component spin site and thus a polar dimer cannot be realized.

For a better understanding of the newly identified ferroelectric spin-Peierls state, it is interesting to compare this with other conventional (non-ferroelectric) spin-Peierls systems. The $H-T$ phase diagrams of spin-Peierls systems are known to be reduced to the universal one when plotted on a plane of reduced temperature versus reduced magnetic field ${ }^{14,23-25}$. In this universal phase diagram, the phase boundary in a low-field regime obeys the following form:

$$
1-\frac{T_{\mathrm{SP}}(H)}{T_{\mathrm{SP}}(0)} \approx \alpha\left[\frac{g \mu_{\mathrm{B}} H}{2 k_{\mathrm{B}} T_{\mathrm{SP}}(0)}\right]^{2}
$$

where $T_{\mathrm{SP}}, g, \mu_{\mathrm{B}}$ and $k_{\mathrm{B}}$ are the spin-Peierls transition temperature, $g$ value, Bohr magneton and Boltzmann constant, respectively.

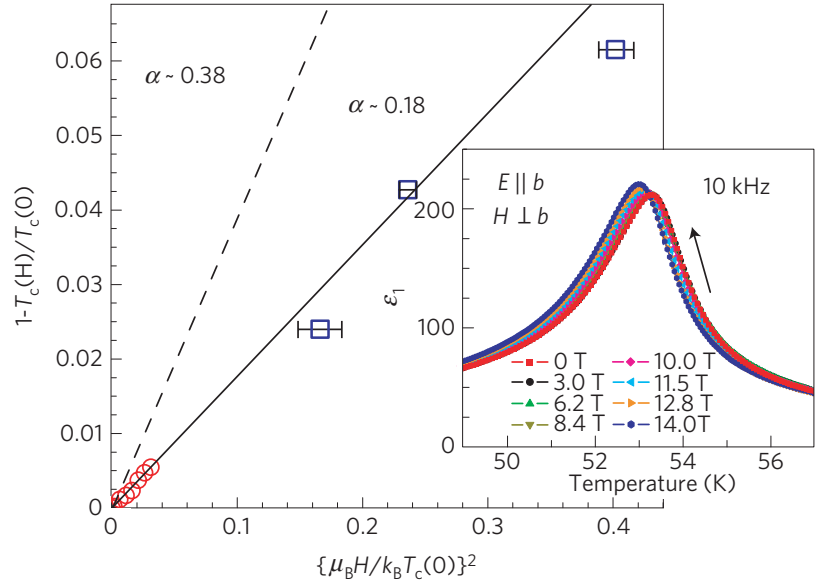

Figure 4 | Magnetic-field dependence of the transition temperature. The transition temperatures are plotted in the form of $1-T_{\mathrm{c}}(H) / T_{\mathrm{c}}(0)$ versus $\left\{\mu_{\mathrm{B}} H / k_{\mathrm{B}} T_{\mathrm{c}}(0)\right\}^{2}$. The squares were obtained from the $\mathrm{M}-H$ curves shown in Fig. 3a, and the open circles were determined from the peak temperature of $\varepsilon_{1}(T)$ at $10 \mathrm{kHz}$ under various magnetic fields shown in the inset. The dashed line represents the theoretical prediction $(\alpha=0.38)$ for the spin-Peierls system ${ }^{23}$, where $\alpha \equiv\left\{1-T_{\mathrm{C}}(H) / T_{\mathrm{C}}(0)\right\} /\left\{\mu_{\mathrm{B}} H / k_{\mathrm{B}} T_{\mathrm{C}}(0)\right\}^{2}$ in a low- $H$ region. The error bars correspond to those shown in Fig. $3 a$.

According to Cross's theory ${ }^{23}$, the value of $\alpha$ is $\sim 0.38$, which is actually in good agreement with the experiments in conventional spin-Peierls systems ${ }^{24,25}$. We plotted $T_{\mathrm{c}}(H)$ of TTF-BA in the form of equation (1) with $g=2$. The result (Fig. 4) demonstrates that in the low-field regime, equation (1) holds even in the ferroelectric spin-Peierls material. However, the value of $\alpha$ turns out to be $\sim 0.18$, significantly smaller than 0.38 . This means that the ferroelectric spin-Peierls state is more robust against a magnetic field.

Why does the spin-Peierls transition in TTF-BA deviate from the conventional framework of spin-Peierls systems? The following two experimental results may provide clues. One is the temperature dependence of the $a_{g}$ mode intensity probing the local $\mathrm{D}^{+} \mathrm{A}^{-}$ dimerization; in Fig. 1d, the $a_{g}$ intensity persists clearly up to $100 \mathrm{~K}\left(\sim 2 T_{\mathrm{c}}\right)$, indicating the dimerization fluctuation well above $T_{\mathrm{c}}$. Note that such fluctuation is not expected far above $T_{\mathrm{c}}$ within the framework of Cross's theory, which is based on the meanfield treatment of spin-phonon coupling. The other clue is the magnitude of the spin gap $\Delta_{\mathrm{s}}(\approx 230 \mathrm{~K})$. Note that $\Delta_{\mathrm{s}} / k_{\mathrm{B}} T_{\mathrm{c}} \approx 4.3$ in TTF-BA, whereas typically $\Delta_{\mathrm{s}}(0) / k_{\mathrm{B}} T_{\mathrm{c}} \approx 2.5$ (ref. 26). Such a large value of $\Delta_{\mathrm{s}} / k_{\mathrm{B}} T_{\mathrm{c}}$ indicates that $T_{\mathrm{c}}$ in TTF-BA is substantially suppressed compared with the mean-field $T_{\mathrm{c}}$ expected from $\Delta_{\mathrm{s}}$. Curiously, unlike the conventional Peierls picture ${ }^{13}, \Delta_{s}(T)$ seems temperature-insensitive in the range $25-53 \mathrm{~K}$ (Supplementary Fig. S1); this peculiarity is readily understood, given that the enhanced dimerization fluctuations would lead to a pseudo-spingap well above $T_{\mathrm{c}}$ in analogy with the pseudo-charge-gap in some charge-Peierls systems ${ }^{27}$. In the light of the strong electron-phonon coupling discussed in the charge-Peierls transition ${ }^{28}$, the two distinct results described above are attributable to strong spinphonon coupling, which may be characteristic of organic donoracceptor-type ferroelectric spin-Peierls system such as TTF-BA.

A key factor for the emergence of magnetically controllable ferroelectricity in TTF-BA is now realized as the spin-Peierls instability. This mechanism is clearly different from the case of hitherto known multiferroics, where the emergence of ferroelectricity relies on the specific magnetic order in spin-frustrated magnets. From the present results, we propose a new guideline for designing symmetric-exchange-driven ferroelectrics, that is, the ferroelectricity induced by low-dimensional instability in quantum spin magnets. The spin-1/2 1D Heisenberg chain composed of binary 
spin sites is the most clear-cut example. Organic donor-acceptor charge-transfer complexes ubiquitously possess the spin-Peierls instability because of the low dimensionality in the stacking of the building blocks ${ }^{29}$, and therefore they may provide a new field of this kind of spin-driven ferroelectricity.

\section{Methods}

Crystal growth. Commercially available TTF and BA were purified by repeated recrystallization and sublimation in a vacuum. An acetonitrile solution of the stoichiometric mixture in a Teflon beaker was slowly evaporated under a stream of argon gas at -5 to $0{ }^{\circ} \mathrm{C}$. The single crystals obtained have a typical dimension of $1.5 \times 1 \times 0.5 \mathrm{~mm}^{3}$.

Pyroelectric and displacement current measurements. The pyroelectric current (or polarization current with sweeping magnetic field) along the $b$ axis was measured under zero electric field after carrying out a poling procedure with a cooling field of $\sim 4.8 \mathrm{kV} \mathrm{cm}^{-1}$. Along the $a$ axis, we could not obtain a reproducible pyroelectric current even though the crystal was cooled under $\sim 8 \mathrm{kV} \mathrm{cm}^{-1}$. The magnetic-field $(H)$ dependence of $P(\| b)$ was measured up to $56 \mathrm{~T}$ in a pulsed magnetic field with a duration of $37 \mathrm{~ms}$, at the International MegaGauss Science Laboratory of the Institute for Solid State Physics, University of Tokyo. The $P-H$ curves $(H \perp b)$ were obtained by integrating the displacement current $\mathrm{d} P / \mathrm{d} t$ as a function of time $t$.

Magnetization measurements. The $M-H$ curve was measured up to $56 \mathrm{~T}$ in a pulsed magnetic field with a duration of $37 \mathrm{~ms}$. The experimental set-up is described, for example, in ref. 30. To attain a good signal-to-noise ratio, we needed a large sample volume and therefore used an assembly of many randomly oriented small crystals of TTF-BA (total weight: $\sim 57.5 \mathrm{mg}$ ).

Infrared reflectivity measurements. To deduce $\varepsilon_{2}$ spectra of the TTF- $a_{g} \nu_{3}$ mode at $1,422 \mathrm{~cm}^{-1}$, the infrared reflectivity data, measured with a Fourier-transform type spectrometer, were fitted with the Lorentz oscillator function (Supplementary Fig. S2). The spectral weight was deduced by the $\omega$-integral of the optical conductivity $\omega \varepsilon_{2}(\omega)$ spectrum.

Received 3 July 2009; accepted 2 December 2009; published online 10 January 2010

\section{References}

1. Kimura, T. et al. Magnetic control of ferroelectric polarization. Nature 426, 55-58 (2003).

2. Hur, N. et al. Electric polarization reversal and memory in a multiferroic material induced by magnetic fields. Nature 429, 392-395 (2004).

3. Cheong, S. W. \& Mostovoy, M. Multiferroics: A magnetic twist for ferroelectricity. Nature Mater. 6, 13-20 (2007).

4. Kenzelmann, M. et al. Magnetic inversion symmetry breaking and ferroelectricity in $\mathrm{TbMnO}_{3}$. Phys. Rev. Lett. 95, 087206 (2005).

5. Arima, T. et al. Collinear to spiral spin transformation without changing the modulation wavelength on ferroelectric transition in $\mathrm{Tb}_{1-x} \mathrm{Dy}_{x} \mathrm{MnO}_{3}$. Phys. Rev. Lett. 96, 097202 (2006).

6. Katsura, H., Nagaosa, N. \& Balatsky, A. V. Spin current and magnetoelectric effect in noncollinear magnets. Phys. Rev. Lett. 95, 057205 (2005).

7. Mostovoy, M. Ferroelectricity in spiral magnets. Phys. Rev. Lett. 96, 067601 (2006).

8. Sergienko, I. A. \& Dagotto, E. Role of the Dzyaloshinskii-Moriya interaction in multiferroic perovskites. Phys. Rev. B 73, 094434 (2006).

9. Sergienko, I. A., Şen, C. \& Dagotto, E. Ferroelectricity in the magnetic E-phase of orthorhombic perovskites. Phys. Rev. Lett. 97, 227204 (2006).

10. Choi, Y. J. et al. Ferroelectricity in an Ising chain magnet. Phys. Rev. Lett. 100, 047601 (2008).

11. Tokunaga, Y. et al. Composite domain walls in a multiferroic perovskite ferrite. Nature Mater. 8, 558-562 (2009).

12. Picozzi, S., Yamauchi, K., Sanyal, B., Sergienko, I. A. \& Dagotto, E. Dual nature of improper ferroelectricity in a magnetoelectric multiferroic. Phys. Rev. Lett. 99, 227201 (2007).
13. Peierls, R. E. Quantum Theory of Solids (Clarendon, 1964).

14. Bulaevskii, L. N., Buzdin, A. I. \& Khomskii, D. I. Spin-Peierls transition in magnetic field. Solid State Commun. 27, 5-10 (1978).

15. Cross, M. C. \& Fisher, D. S. A new theory of the spin-Peierls transition with special relevance to the experiments on TTFCuBDT. Phys. Rev. B 19, 402-419 (1979).

16. Horiuchi, S. \& Tokura, Y. Organic ferroelectrics. Nature Mater. 7, 357-366 (2008)

17. García, P. et al. Crystallographic investigation of temperature-induced phase transition of the tetrathiafulvalene- $p$-bromanil, TTF-BA charge transfer complex. Phys. Rev. B 72, 104115 (2005).

18. Girlando, A., Pecile, C. \& Torrance, J. B. A key to understanding ionic mixed stacked organic solids: tetrathiafulvalene-bromanil (TTF-BA). Solid State Commun. 54, 753-759 (1985).

19. Torrance, J. B. et al. Anomalous nature of neutral-to-ionic phase transition in tetrathiafulvalene-chloranil. Phys. Rev. Lett. 47, 1747-1750 (1981).

20. Tokura, Y. et al. Domain-wall dynamics in organic charge-transfer compounds with one-dimensional ferroelectricity. Phys. Rev. Lett. 63, 2405-2408 (1989).

21. Jacobs, I. S. et al. Spin-Peierls transitions in magnetic donor-acceptor compounds of tetrathiafulvalene (TTF) with bisdithiolene metal complexes. Phys. Rev. B 14, 3036-3051 (1976).

22. Hase, M., Terasaki, I. \& Uchinokura, K. Observation of the Spin-Peierls transition in linear $\mathrm{Cu}^{2+}$ (spin-1/2) chains in an inorganic compound $\mathrm{CuGeO}_{3}$. Phys. Rev. Lett. 70, 3651-3654 (1993).

23. Cross, M. C. Effect of magnetic fields on a spin-Peierls transition. Phys. Rev. B 20, 4606-4611 (1979)

24. Northby, J. A. et al. Field-dependence differential susceptibility studies on tetrathiafulvalene- $\mathrm{AuS}_{4} \mathrm{C}_{4}\left(\mathrm{CF}_{3}\right)_{4}$ : Universal aspects of the spin-Peierls phase diagram. Phys. Rev. B 25, 3215-3225 (1982).

25. Hase, M. et al. Magnetic phase diagram of the spin-Peierls cuprate $\mathrm{CuGeO}_{3}$. Phys. Rev. B 48, 9616-9619 (1993).

26. Orignac, E. \& Chitra, R. Mean-field theory of the spin-Peierls transition. Phys. Rev. B 70, 214436 (2004).

27. Schäfer, J. et al. High-temperature symmetry breaking in the electronic band structure of the quasi-one-dimensional solid $\mathrm{NbSe}_{3}$. Phys. Rev. Lett. 87, 196403 (2001).

28. Monceau, P. Nature of the Peierls transition in charge density wave systems: Strong coupling versus weak coupling. Physica D 216, 167-171 (2006).

29. Torrance, J. B. The difference between metallic and insulating salts of tetracyanoquinodimethane (TCNQ): How to design an organic metal. Acc. Chem. Res. 12, 79-86 (1979).

30. Kindo, K. 100 T magnet developed in Osaka. Physica B 294-295, 585-590 (2001).

\section{Acknowledgements}

We thank N. Nagaosa, M. Imada, R. Kumai, S. Miyahara, S. Watanabe and Y. Takahashi for fruitful discussion. This work was partially supported by Grants-in-Aid for Scientific Research on Innovative Areas (Grant No. 20110003) and Priority Areas 'High Field Spin Science in 100 T' (No. 451) from the MEXT and a Grant-in-Aid for Scientific Research (Grant No. 20340086) from JSPS.

\section{Author contributions}

F.K. and S.H. carried out the dielectric and polarization measurements. F.K. and M.T. carried out the pulsed high-magnetic-field measurements. J.F. carried out the optical study. F.K. carried out the low-field magnetization measurements. S.H., Y.T. and F.K. planned and headed the project. S.H. grew the single crystals for the study. F.K. and Y.T. discussed the magnetic-field effect on $T_{\mathrm{c}}$. F.K. wrote the letter with assistance from Y.T., S.H. and M.T.

\section{Additional information}

The authors declare no competing financial interests. Supplementary information accompanies this paper on www.nature.com/naturephysics. Reprints and permissions information is available online at http://npg.nature.com/reprintsandpermissions Correspondence and requests for materials should be addressed to F.K. 